\title{
Alteraciones en el vinculo materno- infantil: prevalencia, factores de riesgo, criterios diagnósticos y estrategias de evaluación
}

\section{Mother-infant bonding disorders: prevalence, risk factors, diagnostic criteria and assessment strategies}

Bruma Palacios-Hernández

Forma de citar: Palacios Hernández B. Alteraciones en el vínculo materno-infantil: prevalencia, factores de riesgo, criterios diagnósticos y estrategias de evaluación. Rev Univ Ind Santander Salud. 2016; 48(2): $164-176$. DOI: http://dx.doi.org/10.18273/revsal.v48n2-2016001 (c) (1) Ð

\section{RESUMEN}

La interacción temprana entre una madre y su bebé durante el primer año de posparto, se centra en la respuesta emocional materna que determina el vínculo materno-infantil, considerado éste un importante mediador en el desarrollo psicosocial del infante. Alteraciones en esta vinculación temprana se asocian a importantes consecuencias en el infante a nivel cognitivo, social, de salud mental y con riesgo de maltrato, abuso infantil y hasta infanticidio. Los factores de riesgo para una alteración en el vínculo materno-infantil incluyen factores de la madre, del bebé, del embarazo, parto y posparto y del ambiente social. El estudio de los aspectos psicosociales de la relación temprana entre una madre y su infante, ha sido de principal interés en países desarrollados; sin embargo, en Latinoamérica la investigación es aún muy escasa. En la presente revisión se exploran las características del vínculo materno-infantil, sus factores de riesgo y consecuencias negativas más asociadas, así como una identificación de las estrategias de evaluación más usadas en diferentes países. La detección temprana de una problemática del vínculo materno-infantil es una estrategia fundamental para diseñar intervenciones de salud específicas y pertinentes que disminuyan las consecuencias adversas y promuevan el bienestar de la diada madre-bebé y su ámbito familiar.

Palabras claves: Vínculo materno-infantil, salud mental materna, Latinoamérica, instrumentos de medición.

\footnotetext{
ABSTRACT

The early relationship between a mother and her baby during the first year after childbirth is focused in the maternal affective response which is the base of the mother-infant bonding, considered an important mediator in the psychosocial child development. Early bonding difficulties are associated to

1. Universidad Autónoma del Estado de Morelos. México.

Correspondencia: Bruma Palacios-Hernández. Dirección: Pico de Orizaba 1, Col. Los Volcanes. C.P. 62350. Cuernavaca, Morelos. México. Correo electrónico: brumaph@hotmail.com Teléfono: (52) 7773297970. 
negative consequences in the infant's cognitive, social and mental health development and a high risk for child maltreatment, abuse and infanticide. Risk factors for a mother-infant bonding disorder include aspects related to the mother, the baby, pregnancy, childbirth and postpartum and the social environment. The study of the psychosocial aspects of the early relationship between a mother and her baby is of main interest in developed countries, nevertheless the research on this topic in LatinAmerica is still very limited. In the present review it is explored the characteristics of a mother-infant bonding, its main risk factors and most relevant negative consequences, as well as the most popular assessment strategies used among the countries. The early detection of a mother-infant bonding disorder is a decisive strategy to allow the design of specific and relevant health interventions that can reduce the adverse consequences and promote the wellbeing of the mother-infant dyad and its family.

Keywords: Mother-infant bonding, maternal mental health, LatinAmerica, assessment measures.

\section{INTRODUCCIÓN}

El procrear un bebé no garantiza una respuesta maternal en la mujer que permita la supervivencia del infante y su desarrollo psicosocial. Es necesaria una vinculación afectiva entre la madre y su recién nacido denominada vínculo materno-infantil que incluya una relación recíproca, favorable y significativa entre ambos ${ }^{1}$. Para Brockington ${ }^{2}$ la relación entre una madre y su bebé es uno de los procesos psicológicos más importantes que se desarrollan después del parto. Si la vinculación materna con el bebé se altera puede generar consecuencias negativas de gran importancia para el bienestar psicosocial, principalmente del infante con efectos negativos de mediano y largo plazo en su desarrollo ${ }^{3}$. Algunas de las consecuencias negativas en el infante incluyen afectaciones en la adaptación y autoregulación emocional $^{4,5}$ así como en el temperamento infantil y la regulación del estrés ${ }^{6}$, el desarrollo cognitivo ${ }^{7}$ y del lenguaje ${ }^{8}$, en la adaptación social ${ }^{3,9}$, el desarrollo funcional y biológico cerebral ${ }^{10}$, el tipo de apego infantil $^{11}$ que condicionará sus interacciones sociales y el desarrollo de psicopatología en la infancia y adolescencia $^{12}$. En casos de vínculos materno-infantil gravemente alterados, el bebé es expuesto a la agresión materna, con riesgo de negligencia grave, abuso, y maltrato infantil ${ }^{3}$, con consecuencias duraderas físicas, emocionales y psicopatológicas ${ }^{13}$ y en casos extremos riesgo de infanticidio $^{14}$ seguido frecuentemente por el suicidio materno ${ }^{15,16}$. A su vez, existe evidencia de que una vinculación temprana favorable entre la madre y su bebé puede disminuir el impacto de factores adversos en el desarrollo infantil, como por ejemplo la reducción del efecto negativo que una exposición in utero de cortisol (producida por estrés materno) tiene asociado a un desarrollo cognitivo alterado en el infante ${ }^{17}$.

La confirmación de consecuencias negativas en el desarrollo psicosocial de infantes que han experimentado un trastorno en su relación afectiva temprana con su madre, han impulsado el estudio de la vinculación materno-infantil en los últimos 30 años. En la presente revisión exploraremos los hallazgos científicos más relevantes sobre la prevalencia de este tipo de trastornos, los factores de riesgo asociados, criterios para su diagnóstico clínico y algunas de las estrategias de evaluación más utilizadas a lo largo del mundo. Asimismo, presentamos una breve descripción del estado de investigación en Latinoamérica sobre este campo y sus principales retos.

\section{DEFINICIÓN Y EVOLUCIÓN DEL CONCEPTO}

Broad, et al. ${ }^{18}$ describieron que dada la indefensión y vulnerabilidad del recién nacido, la vinculación afectiva entre madre y bebé representa una interacción social fundamental para la supervivencia del bebé, cuya respuesta materna es facilitada por mecanismos hormonales, de recompensa cerebral y reconocimiento sensorial. El contacto piel con piel y el amamantamiento promueven la liberación de oxitocina en las madres después del parto que tranquiliza a la madre y la hace más receptiva hacia

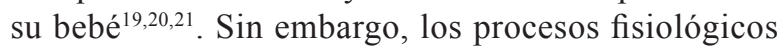
no garantizan una respuesta maternal favorable hacia el recién nacido pues ésta requiere adicionalmente de conductas y respuestas afectivas, desarrolladas en circunstancias específicas no determinadas inherentemente por un patrón conductual instintivo ${ }^{1}$. Stern, et al. ${ }^{22}$ sugirieron que la mujer requiere desarrollar una nueva configuración psicológica que le permita la reorganización de su identidad para asumir un nuevo rol como madre. Winnicot ${ }^{23}$ llamó "preocupación primaria maternal" a un estado psicológico durante el embarazo dirigido al infante aún no nacido, que será fundamental para una adecuada vinculación afectiva entre madre y recién nacido ${ }^{3}$. 
El desarrollo de John Bowlby ${ }^{24,25}$ y sus futuros colaboradores $^{26}$ de una teoría del apego, atrajo la atención hacia el estudio de las relaciones tempranas entre los progenitores y sus infantes. Para Bowlby ${ }^{25}$ un comportamiento de apego es un aspecto especifico $y$ circunscrito de la relación entre un infante y su cuidador principal que tiene como objetivo el hacer sentir al bebé seguro, a salvo y protegido. El apego será una conducta fundamental que permitirá la supervivencia del infante ${ }^{24}$ y protegerá al bebé de cualquier situación que ponga en riesgo su bienestar, brindándole una figura que actúe como una base segura en la cual resguardarse en caso de ser necesario y también fomente la exploración de su medio ambiente. El proceso será exitoso, si los padres son sensibles en identificar y entender las necesidades y el estado emocional de su bebé, para lograr disminuir la frustración y ansiedad del bebé originada por la interacción con el medio ambiente ofreciendo una base segura cuando la requiera. Esta conducta evolucionará en patrones de conducta organizados que se mantendrán estables a lo largo de la vida del infante afectando su desarrollo y las interacciones sociales futuras ${ }^{18,24,26}$.

Posteriormente el interés se centró en las interacciones más tempranas entre madre y bebé, cuando el bebé aún es incapaz de reaccionar de forma organizada con una conducta de apego hacia su cuidador. Esta temprana interacción fue definida por Klaus y Kennell ${ }^{27}$ como "vinculación" entre una madre y su bebé, centrándose en la respuesta emocional materna. Aunque los conceptos de apego y vinculación materno-infantil analizan la diada madre-bebé, no son sinónimos pues se centran en diferentes procesos y momentos de la relación. La vinculación materno-infantil refiere a $l a$ relación temprana entre una madre y su infante cuya principal característica es la respuesta emocional materna hacia su bebé $e^{3,28}$ durante el primer año de vida del bebé ${ }^{29}$. El apego es un proceso posterior centrado en la reacción del infante ya organizada en un patrón estable dirigida hacia el cuidador principal ${ }^{3,28,30}$. La cercanía de ambos conceptos suele generar confusión sobre todo cuando la definición del vínculo maternoinfantil aún muestra una evolución conceptual, lo cual representa un reto para su evaluación y estudio.

\section{CRITERIOS DIAGNÓSTICOS}

Hasta la fecha no existe una alteración o trastorno en el vínculo materno-infantil reconocido como desorden psicológico en ninguna de las dos clasificaciones diagnósticas más usadas en salud mental: el Manual
Diagnóstico y Estadístico de Trastornos Mentales $[\mathrm{DSM}-5]^{31}$ y la Clasificación Estadística Internacional de Enfermedades de la Organización Mundial de la Salud [ICD-10] $]^{32}$.

El DSM-5 $5^{31}$ únicamente identifica trastornos del apego observado en infantes desde los 9 meses hasta los 9 años, centrándose en la sintomatología visible en el niño y no en el padre (Trastorno de apego reactivo, Código F94.1). El ICD-10 ${ }^{32}$ cuenta con un diagnóstico semejante al DSM-5, referido como trastorno de vinculación en la infancia reactivo (Código F94.1). Lo más cercano a una alteración en el vínculo materno-infantil centrada en la conducta del progenitor se identifica en el DSM-5 que propone la evaluación clínica de problemas de relación entre un padres e hijos (Código V61.20/Z62.820) como una condición que puede ser de interés clínico sin considerarlo un trastorno mental. El problema entre un hijo y su cuidador principal, debe generar un efecto negativo en la salud del infante asociados a maltrato infantil o negligencia, con consecuencias médicas y psicológicas significativas para el hijo (p.e. abuso físico infantil, negligencia infantil o abuso psicológico infantil) sin aportar criterios diagnósticos para su diagnóstico clínico. Lo más cercano al reconocimiento de un desorden relacionado con el vínculo maternoinfantil se encuentra en la Clasificación Diagnóstica de Salud Mental y Trastornos del Desarrollo de la Infancia y la Temprana Infancia (Cero a Tres) de la Asociación Americana de Psicología [DC:0-3R $]^{33}$. El DC:0-3R permite diagnosticar un involucramiento excesivo, deficiente, ansioso, hostil o abusivo de los padres con sus infantes.

Estudios ${ }^{3,34}$ han descrito que una alteración del vínculo materno-infantil, puede presentarse en diversos niveles desde el retraso, la ambivalencia o carencia de respuesta afectiva materna, hasta el rechazo materno, o ira y hostilidad hacia el bebé, asociados en sus formas extremas con el maltrato y abuso infantil. Basados en evidencia científica y amplia experiencia clínica Brockington ${ }^{3}$, Aucaump \& Fraser $^{35}$, sugirieron criterios diagnósticos específicos para identificar un trastorno en el vínculo materno-infantil (Tabla 1). Ante la carencia de una definición internacionalmente aceptada y basado en la revisión de las aportaciones de investigaciones previas, una alteración en el vínculo materno-infantil puede definirse como: una alteración en la respuesta emocional materna hacia el bebé presente en la interacción temprana entre ambos posterior al parto y durante el primer año del posparto. 
Tabla 1. Criterios para diagnosticar un desorden en el vínculo materno-infantil (Brockington, Aucamp \& Fraser, 2006 $6^{35}$ )

\begin{tabular}{lll}
\hline Tipo Descripción & Criterios
\end{tabular}

\section{* Los criterios A hasta el D deben ser positivos:}

Estas madres experimentan un retraso en el desarrollo, ambivalencia o pérdida de la respuesta afectiva maternal hacia su bebé. a. La madre expresa decepción sobre su respuesta emocional (p.e. no tiene ninguna emoción hacia su bebé o se siente extraña o distante de su bebé- este "no es su bebé", o siente como si estuviera cuidándoselo a alguien más.

Desorden Leve cumplen.

c. El desorden ha durado por lo menos una semana.

d. Las emociones son percibidas por la madre como causas de estrés y han ocasionado que busque ayuda de su familia o de profesionales.

\section{Amenaza de Rechazo}

a. El bebé no es deseado por la madre en el momento de la evaluación y el deseo materno se orienta hacia una transferencia temporal de

Estas madres carecen de una respuesta emocional positiva hacia su bebé, además de que han desarrollado un deseo por

Desorden Severo (Rechazo del bebé) renunciar o deshacerse del mismo.

Dos tipos: Amenaza de Rechazo o Rechazo establecido cuya principal diferencia es la permanencia del deseo de deshacerse del bebé. su cuidado.

b. Las madres carecen de una significativa aversion hacia el bebé y NO han experimentado un deseo para que el bebé "desaparezca".

\section{Rechazo establecido}

Los criterios A, B o C son necesarios para el diagnóstico:

a. La madre expresa desagrado, resentimiento u odio por su bebé. A veces es manifestado con expresiones como: "Deseo que hubiese nacido muerto", o "Este bebé me ha arruinado mi vida".

b. La madre expresa su deseo por deshacerse del cuidado de su bebé de forma permanente.

c. La madre ha experimentado un deseo de que su bebé desaparezcap.e. deseo de que el bebé sea robado, o que sea víctima del síndrome de muerte súbita.

\section{Ansiedad centrada en el infante.}

Identifica la presencia de ansiedad materna relacionada con el bebé. Deben especificarse dos niveles: leve y severo.

a. Ansiedad Leve: La madre refiere sentirse ansiosa cuando está sola con el infante.

b. Ansiedad severa: La ansiedad reduce el contacto con el bebé.

\section{Ira Patológica: 3 niveles- leve,moderada y severa.}

a. Leve:

- La madre ha perdido su autocontrol verbal en al menos dos ocasiones (gritado o insultado al bebé).

Además de los diagnósticos previos de desorden leve $\mathrm{y}$ amenaza de rechazo o rechazo

Diagnósticos Adicionales establecido, pueden hacerse diagnósticos complementarios de: Ansiedad centrada en el infante y/o ira patológica.
- La madre no ha expresado su ira en otra forma.

Nota: La ira experimentada internamente y controlada con dificultad, no califica. Una madre que pierde el control de forma verbal en una ocasión es considerado un comportamiento normal.

b. Ira moderada:Además de calificar para criterios de ira patológica leve:

- La madre experimenta impulsos de hacerle daño al bebé (p.e. asfixiarlo, tirarlo o golpearle),

- O han existido episodios de abuso, como sacudir violentamente la carreola del bebé.

Nota: Debe discriminarse entre impulsos agresivos maternos y aquellos que madres con un Trastorno Obsesivo-Compulsivo experimentan como parte del desorden.

c. Ira Severa:

- Además de la pérdida de control verbal materno, o de los impulsos de hacerle daño al bebé (criterios $a+b)$, al menos debe existir un episodio de claro abuso infantil. 


\section{FACTORES DE RIESGO}

El parto y el posparto suelen ser experiencias estresantes para una mujer que exigen esfuerzos físicos, psicológicos y emocionales extraordinarios para atender las demandas que el cuidado de un recién nacido requiere. Durante la temprana interacción madrebebé, ciertos factores adicionales al estrés propio del posparto, pueden alterar de forma negativa el proceso. Los factores de riesgo más frecuentemente asociados a una alteración vincular incluyen factores de la madre, del bebé, del embarazo, el parto y el posparto y del ambiente social.

Dentro de los factores relacionados con la madre, se encuentran la presencia de psicopatologia materna ${ }^{2,36,37}$, experiencias negativas pasadas con los propios padres $^{30,38,39}$, edad joven materna ${ }^{1}$, bajo nivel de educación ${ }^{40}$, bajo nivel socioeconómico ${ }^{41}$, embarazo no deseado $^{3,9,30,42}$ y falta de apego prenatal con el feto ${ }^{3,43}$.

Entre los factores relacionados con el bebé más relevantes, se encuentran condiciones médicas severas sufridas por el bebé, incluyendo anormalidades, prematuridad, bajo peso al nacer o un temperamento difícil ${ }^{44-46}$. El orden de nacimiento del bebé también es relevante. Se ha identificado que madres primerizas reportan mayores dificultades en la interacción temprana, ya sea por la inexperiencia o por altos niveles de ansiedad y preocupaciones sobre el cuidado infantil ${ }^{47}$, así como presentan un mayor retraso de la respuesta emocional positiva hacia el bebé ${ }^{48}$ y también descripciones más negativas de sus bebés ${ }^{49}$ que madres multíparas. El sexo femenino del bebé ha sido también un factor negativo para la vinculación temprana materno-infanti $1^{50,51}$, asociado a aspectos socioculturales en donde existen mayores desventajas relacionadas con el género observado en ciertos países ${ }^{52}$.

Procesos propios del embarazo, el parto y el posparto también se encuentran asociados a alteraciones del vínculo materno-infantil. Uno de los más relevantes es la lactancia materna, identificada tanto como un factor de protección como de riesgo. La lactancia materna se ha asociado de forma positiva al facilitar una mayor respuesta y sensibilidad materna hacia el bebés3 y facilitar la vinculación emocional ${ }^{54}$. Sin embargo, la lactancia materna también puede transformarse en un factor de riesgo siendo considerada la cesación temprana o ausencia como un síntoma temprano de dificultades en la vinculación madre-bebés5. La no lactancia también ha mostrado una asociación cercana con la salud mental materna particularmente afectada por la depresión y ansiedad ${ }^{55-57}$. Estudios han reportado una interrelación entre la depresión materna, la no lactancia y dificultades en el vínculo materno-infanti $1^{49}$. El tipo y la experiencia del embarazo y del parto, sobre todo si fueron experimentados como una experiencia traumática, es otro factor de riesgo para la vinculación afectiva entre una madre y su bebé $e^{3,30,58,59}$. Finalmente el medio ambiente social con un bajo apoyo social y la falta de apoyo o problemas con la pareja son factores de riesgo para una alteración vincular temprana ${ }^{1,30,45,59}$.

\section{PREVALENCIA}

El principal reto para estimar la prevalencia de alteraciones en el vínculo materno-infantil entre diferentes grupos y países es la heterogeneidad de instrumentos y estrategias de evaluación, así como de diferentes momentos de estudio durante el primer año del posparto. La identificación de la prevalencia de alteraciones en el vínculo materno-infantil ha sido hecha en su mayoría en países desarrollados. Evidencia científica ha identificado una clara asociación entre la salud mental materna con la calidad de la interacción temprana entre madre y bebé, el bienestar del bebé y el desarrollo psicosocial con consecuencias relevantes a largo plazo ${ }^{2,5,37}$. Estudios que han utilizado los mismos instrumentos de evaluación ${ }^{60-62}$ confirman una mayor prevalencia de trastornos del vínculo materno-infantil (22-67\%) en madres con diversos trastornos mentales ${ }^{5,35,60,61,63}$ que en madres de población general $(7-9 \%)^{64-66}$.

La evidencia en madres de países en vías de desarrollo y en particular de Latinoamérica es aún escasa. Una revisión de literatura en el tema de vínculo maternoinfantil reportó 2597 artículos publicados hasta el 11 de enero del 2016 (años 1962-2016) con un incremento significativo en los últimos 10 años $(\mathrm{n}=1241)$ que representan casi la mitad de la producción científica total (Base de datos: SCOPUS; palabras claves: mother-infant bonding (OR) mother-infant early relationship (OR) mother-infant interaction (OR) mother-infant bonding disorder). Estados Unidos de América $(\mathrm{n}=1128)$, el Reino Unido $(\mathrm{n}=217)$ y Canadá $(\mathrm{n}=158)$ son los países que más estudios han publicado sobre la temática identificándose una muy baja producción científica en Latinoamérica con sólo 77 artículos realizados en esta región (3\% de la producción mundial) en el periodo evaluado. Excluyendo a los estudios que fueron realizados en poblaciones externas 
a la region, se identificó producción científica en sólo 10 países de Latinoamérica siendo Brasil $(n=37$, $48.1 \%)$, Chile $(n=12,15.6 \%)$ y México $(n=9,11.7 \%)$ los principales países que reportan investigación en el campo.

Estos datos sugieren un retraso significativo en la producción científica, sobre la vinculación temprana entre una madre y su bebé en estudios con poblaciones latinoamericanas, lo cual dificulta el conocimiento sobre la dimensión de alteraciones en este proceso así como de los factores de riesgo relevantes en estos países. Por otra parte, aunque la salud materna fue uno de los 8 objetivos de desarrollo del Milenio estipulados por la Organización de las Naciones Unidas (ONU), no se incorporó la atención a la salud mental materna como parte de las prioridades estratégicas de mejoramiento de la salud de las madres o los infantes ${ }^{67}$. La ONU ha identificado en 2015 desigualdades significativas en Latinoamérica en: el acceso y uso de servicios de salud reproductiva entre las zonas urbanas y rurales, una alta prevalencia de embarazos en adolescentes, un incremento de más mujeres que hombres en hogares pobres y una desigualdad en la disponibilidad de información relacionada con salud materna que dificultan el establecimiento de prioridades de salud ocasionados por una disparidad sanitaria entre países y entre los grupos vulnerables por su situación social, económica, de educación, edad o lugar de residencia ${ }^{67}$. Estos determinantes sociales constituyen riesgos para la salud mental de la madre y su vinculación temprana con su bebé desconociendo en gran medida su efecto a corto y largo plazo en poblaciones latinoamericanas.

\section{EVALUACIÓN}

Las estrategias de evaluación que permiten evaluar e investigar las dificultades en la interacción temprana entre una madre y su bebé se pueden clasificar en a) autoreportes, b) entrevistas clínicas especializadas o listas de puntajes con evaluadores externos (enfermeras o profesionistas de la salud mental) y, c) evaluaciones interactivas y/o de observación directa para evaluar a la diada madre-bebé videograbadas u observadas en escenarios clínicos o en casa. La evaluación más usada en ámbitos clínicos y de investigación es el autoreporte por su fácil uso e interpretación identificando de la fuente principal la respuesta emocional materna hacia su bebé. Los autoreportes más usados a nivel mundial, detallados en la Tabla 2, muestran que sólo un instrumento ha sido validado en Brasil ${ }^{68}$ lo cual refleja una escasez de instrumentos diseñados y validados en Latinoamérica (y ausencia de instrumentos en español) ${ }^{69}$ que dificulta la detección temprana de este tipo de trastornos y la comprensión de la dimensión de la problemática y los factores específicos involucrados entre los diferentes grupos poblacionales.

El uso de entrevistas clínicas, ha sido menos frecuente al requerir mayores recursos, pero resulta fundamental para confirmar el diagnóstico de una alteración en el vínculo materno-infantil. Dentro de las entrevistas especializadas más usadas se encuentran la Entrevista Birmingham de Salud Mental Materna [BMMHI] ${ }^{62}$ o su sexta edición de reciente diseño ahora denominada Entrevista Stafford (Brockington, et al. 2014, en prensa) las cuales cuentan con una sección para evaluar la relación temprana entre la madre y el bebé y criterios diagnósticos para descartar o confirmar un trastorno vincular. Otras entrevistas para estos mismos fines son el Inventario Yale sobre pensamientos y acciones paternas $[\text { YIPTA }]^{70}$ y la entrevista semiestructurada de Evaluación Contextual de la Experiencia Maternal $[\mathrm{CAME}]^{71}$.

La mayoría de los estudios han utilizado sólo autoreportes o entrevistas para evaluar dificultades vinculares. Sin embargo, esto conlleva el riesgo de una mayor probabilidad de simulación en las respuestas maternas y de sesgo en los datos ${ }^{3}$. Por ello, se sugiere sumar ambas estrategias y si se cuenta con los recursos suficientes, incluir observaciones directas que cuentan con un mayor nivel de confiabilidad (p.e. Codificación Interactivo del Comportamiento [Coding Interactive Behavior-CIB] $]^{72}$, Escala Bethlem de Interacción madre-infante [Blethlem Mother-Infant Interaction Scale-BMIS] $]^{73}$, Care-Index ${ }^{74}$ ). En caso de evaluación por medio de entrevistas clínicas y de observaciones directas, se sugiere usar a dos evaluadores quienes independientemente realicen un dictamen sobre la presencia o ausencia de una alteración del vínculo materno-infantil y posteriormente se calcule el grado de fiabilidad inter-evaluador y se obtengan diagnósticos consensuados ${ }^{61,75,76}$. Ésta ha resultado ser una estrategia útil para eliminar el sesgo subjetivo de la evaluación e incrementar el grado de confiabilidad de los resultados obtenidos. 
Alteraciones en el vínculo materno-infantil: prevalencia, factores de riesgo, criterios diagnósticos y estrategias de evaluación

Tabla 2. Autoreportes que evalúan la respuesta emocional maternal hacia su bebé y su interacción temprana.

\begin{tabular}{|c|c|c|c|c|c|c|}
\hline Autores & $\begin{array}{c}\text { Nombre/ } \\
\text { *País de Origen }\end{array}$ & Objetivo de evaluación & $\begin{array}{c}\text { Población en que fue } \\
\text { validado }\end{array}$ & Confiabilidad & Características & Validación en otros países \\
\hline $\begin{array}{l}\text { Kumar, Robson, } \\
\& \text { Smith, } 1984^{77}\end{array}$ & $\begin{array}{l}\text {-Ajuste Materno y Actitudes } \\
\text { Maternas } \\
\text { [MAMA] (Maternal Adjustment } \\
\text { and Maternal Attitudes) } \\
\text { *Reino Unido }\end{array}$ & $\begin{array}{l}\text {-Patrones de cambio } \\
\text { relacionados con el ajuste } \\
\text { materno, la relación } \\
\text { marital y las actitudes } \\
\text { hacia el bebé }\end{array}$ & $\begin{array}{l}\mathrm{N}=99 \\
\text { Madres primerizas }\end{array}$ & $\begin{array}{l}\text {-Índices adecuados en } \\
\text { los métodos de test- } \\
\text { retest y de división } \\
\text { por mitades }\end{array}$ & $\begin{array}{l}\text { - } 60 \text { ítems. } \\
-5 \text { subescalas } \\
-2 \text { versiones: embarazo y } \\
\text { posparto } \\
\text { - Versión Posparto: subescala de } \\
\text { ajuste materno y actitudes hacia } \\
\text { el bebé ( } 12 \text { ítems), escala de } \\
\text { respuesta de } 4 \text { puntos }\end{array}$ & $\begin{array}{l}\text { - } \text { Portugal }^{78} \\
\text { - } \text { Suecia }^{79} \\
\text { - } \text { Grecia }^{80} \\
\text { Usado en muestras de: } \\
\text { - Estados Unidos de } \\
\text { América } \\
\text { - Finlandia }\end{array}$ \\
\hline Muller, $1994^{83}$ & $\begin{array}{l}\text { Inventario de Apego materno } \\
{[\mathrm{MAI}] \text { (The Maternal }} \\
\text { Attachment Inventory) } \\
\text { *Estados Unidos de América }\end{array}$ & $\begin{array}{l}\text {-Apego emocional } \\
\text { materno en la relación de } \\
\text { una mujer hacia su bebé }\end{array}$ & $\begin{array}{l}\mathrm{N}=196 \\
\text { Madres evaluadas al mes } \\
1(\mathrm{~T} 1), 4(\mathrm{~T} 2) \text { y } 8(\mathrm{~T} 3) \\
\text { de posparto }\end{array}$ & $\begin{array}{l}\text { Coeficiente de Alfa de } \\
\text { Conbrach: } \\
\\
\alpha=0.85 \text { (T1) } \\
\alpha=0.76 \text { (T2) } \\
\alpha=0.85 \text { (T3) }\end{array}$ & $\begin{array}{l}\text { - } 26 \text { ítems con una escala tipo } \\
\text { Likert de } 4 \text { opciones de respuesta } \\
\text { (frecuencia de actividades } \\
\text { maternas o emociones) }\end{array}$ & $\begin{array}{l}\text {-Japón } \\
\text {-Korea, } \\
\text {-Brasi }{ }^{88} \\
\text {-China }{ }^{87} \\
\\
\text { Usado en muestras de: } \\
\text {-Australia } \\
\text {-Jordania }\end{array}$ \\
\hline $\begin{array}{l}\text { Warner, Appleby, } \\
\text { Whitton, \& } \\
\text { Faragher, } 1997^{89}\end{array}$ & $\begin{array}{l}\text {-Cuestionario de Actitudes } \\
\text { Maternas } \\
{[\mathrm{MAQ}] \text { (Maternal Attitudes }} \\
\text { Questionnaire) } \\
\text { *Reino Unido } \\
\end{array}$ & $\begin{array}{l}\text {-Pensamientos maternos } \\
\text { sobre: cambio de rol, } \\
\text { expectativas robre } \\
\text { maternidad y como madre } \\
\text { en el posparto }\end{array}$ & $\begin{array}{l}\mathrm{N}=483 \\
\text { Madres de programas de } \\
\text { tamizaje en } 2 \text { Unidades } \\
\text { de Maternidad }\end{array}$ & $\begin{array}{l}\text { Coeficiente Alfa de } \\
\text { Conbrach: } \\
\alpha=0.84\end{array}$ & $\begin{array}{l}-14 \text { ítems con una escala de tipo } \\
\text { Likert de } 4 \text { opciones de respuesta } \\
-4 \text { items identifican la respuesta } \\
\text { maternal hacia el bebé y la } \\
\text { maternidad }\end{array}$ & Ninguna identificada \\
\hline $\begin{array}{l}\text { Condon \& } \\
\text { Corkindale, } \\
1998^{29}\end{array}$ & $\begin{array}{l}\text { Escala de Apego materno } \\
\text { postnatal. } \\
\text { [MPAS] (Maternal Postnatal } \\
\text { Attachment Scale) } \\
\text { *Australia }\end{array}$ & -Apego madre-infante & $\begin{array}{l}\mathrm{N}=200 \\
\text { Madres evaluadas al mes } \\
1 \text { (T1), } 4 \text { (T2) y } 8 \text { (T3) } \\
\text { de posparto }\end{array}$ & $\begin{array}{l}\text { Coeficiente Alfa de } \\
\text { Cronbach: } \\
\begin{array}{ll}\alpha=0.78 \text {. (T1) } \\
\alpha=0.79 \text { (T2) } \\
\alpha=0.78 \text { (T3) }\end{array}\end{array}$ & $\begin{array}{l}-19 \text { ítems con una escala tipo } \\
\text { Likert de } 5 \text { puntos }(1=\text { apego } \\
\text { débil, } 5=\text { apego fuerte }) \\
-3 \text { subescalas: calidad del apego, } \\
\text { ausencia de hostilidad, y placer en } \\
\text { la interacción }\end{array}$ & $\begin{array}{l}\text {-Estados Unidos de } \\
\text { América }^{90} \\
\text {-Italia } \\
\text {-Bélgica }^{96} \\
\text { Usado en muestras de: } \\
\text {-Reino Unido }\end{array}$ \\
\hline $\begin{array}{l}\text { Nagata, et al., } \\
2000^{93}\end{array}$ & $\begin{array}{l}\text { Sin nombre especificado } \\
\text { *Japón }\end{array}$ & $\begin{array}{l}\text {-Apego materno hacia } \\
\text { sus hijos y los factores } \\
\text { asociados al mismo }\end{array}$ & $\begin{array}{l}\mathrm{N}=417 \\
\text { Madres de la Unidad } \\
\text { Obstétrica de un Hospital }\end{array}$ & $\begin{array}{l}\text { Coeficiente Alfa de } \\
\text { Cronbach: } \\
\alpha=0.80\end{array}$ & $\begin{array}{l}\text { - } 21 \text { ítems con una escala tipo } \\
\text { Likert de } 4 \text { opciones } \\
-3 \text { subescalas: aceptación del } \\
\text { bebé, ansiedad enfocada al bebé, } \\
\text { involucramiento con el bebé }\end{array}$ & Sin datos \\
\hline
\end{tabular}

Brockington, et al., $2001^{60}$

Test del Vínculo en el Posparto
$[\mathrm{PBQ}]$ (Postpartum Bonding
Questionnaire)
*Reino Unido

Brockington, Fracer, \& Wilson, Fraser,

$\begin{array}{ll} & \mathrm{N}=104 \\ \text {-Trastornos en el vínculo } & \begin{array}{l}\text { Madres de población } \\ \text { materno-infantil }\end{array} \\ \text { general, deprimidas y de } \\ \text { bebés con anormalidades }\end{array}$

-Correlación Pearson: Factor 1: 0.95 Factor 2: 0.95 Factor 3: 0.93 Factor 4: 0.77
-25 ítems con escala tipo Likert -Alemania ${ }^{65}$ con 5 opciones de respuesta. $\quad$-Holanda ${ }^{94}$ -4 factores: vínculo afectado, $\quad-$ China $^{63}$ rechazo y odio, ansiedad sobre $\quad$ - Bélgica ${ }^{66}$ el cuidado del bebé y riesgo $\quad$-Noruega ${ }^{95}$ -Japón ${ }^{85,96}$ -España ${ }^{97}$

Usado en muestras de: - Suecia $^{64}$

-Bangladesh ${ }^{51}$ -Estados Unidos de $\mathrm{N}=125$ $\begin{array}{lll}\text {-Validar el PBQ en otras } & \begin{array}{l}\text { Madres con trastornos } \\ \text { muestras de madres }\end{array} & \text { No especificadiátricos en el }\end{array}$ posparto

-25 ítems y 4 factores del 2001 -Puntos de corte mejorados: a) Subescalas: $1(\geq 12), 2(\geq 13), 3 \quad$ América $^{98}$ $(\geq 10), 4(\geq 2)$

b) Punto de corte con puntaje total: para identificar cualquier tipo de trastorno vincular $(\geq 26)$ y para trastornos severos $(\geq 40)$

\begin{tabular}{|c|c|c|c|c|c|c|}
\hline $\begin{array}{l}\text { Taylor, Atkins, } \\
\text { Kumar, Adams, } \\
\& \text { Glover, } 2005^{28}\end{array}$ & $\begin{array}{l}\text { Escala de vínculo madre-infante } \\
\text { [MIBS] (Mother-to-infant } \\
\text { Bonding Scale) } \\
\text { *Reino Unido }\end{array}$ & $\begin{array}{l}\text {-Emociones maternas } \\
\text { hacia su recién nacido }\end{array}$ & $\begin{array}{l}\mathrm{N}=162 \\
\text { Madres evaluadas a los } \\
3 \text { días de posparto en un } \\
\text { Hospital de Maternidad }\end{array}$ & $\begin{array}{l}\text { Coeficiente de Alfa de } \\
\text { Cronbach: } \\
\alpha=0.71\end{array}$ & $\begin{array}{l}-8 \text { ítems con una escala tipo likert } \\
\text { de } 4 \text { opciones de respuesta } \\
\text {-Lista de emociones maternas } \\
\text { positivas y negativas hacia } \\
\text { su bebé }\end{array}$ & $\begin{array}{l}\text {-Portugal }{ }^{100} \\
\text {-Bélgica } \\
\text {-Francia } \\
\text {-Japón } \\
\end{array}$ \\
\hline $\begin{array}{l}\text { Hall \& } \\
\text { Papageorgiou, } \\
2005^{105}\end{array}$ & $\begin{array}{l}\text { Cuestionario de Pensamientos } \\
\text { negativos en Posparto [PNTQ] } \\
\text { (Postnatal Negative Thoughts } \\
\text { Questionnaire) } \\
\text { *Reino Unido }\end{array}$ & $\begin{array}{l}\text {-Pensamientos negativos } \\
\text { de la madre durante el } \\
\text { posparto }\end{array}$ & $\begin{array}{l}\mathrm{N}=181 \\
\text { Madres con bebés }(0 \text { a } 7 \\
\text { meses) del Servicio de } \\
\text { Salud Nacional del Reino } \\
\text { Unido }\end{array}$ & $\begin{array}{l}\text { Coeficiente de Alfa de } \\
\text { Cronbach: } \\
\alpha=0.78\end{array}$ & $\begin{array}{l}\text { - Escala de } 3 \text { respuestas ( } 0 \text { a } 3 \text { ) } \\
-2 \text { factores de vínculo materno- } \\
\text { infantil: } \\
\text { 1) Estimación de los } \\
\text { pensamientos, emoción y } \\
\text { situación ( } 9 \text { items) } \\
\text { 2) Pensamientos maternos sobre } \\
\text { el bebé y la maternidad ( } 8 \text { ítems) }\end{array}$ & Sin datos \\
\hline $\begin{array}{l}\text { Høivik, } \\
\text { Burkeland, } \\
\text { Linaker, \& Berg- } \\
\text { Nielsen, 2013 }\end{array}$ & $\begin{array}{l}\text { Escala de interacción madre- } \\
\text { bebé [MABISC] } \\
\text { *Desarrollado en Reino Unido y } \\
\text { usado en Noruega }\end{array}$ & $\begin{array}{l}\text {-Consistencia interna, } \\
\text { estabilidad, componentes } \\
\text { principales y validez } \\
\text { convergentes de la Escala } \\
\text { de } 10 \text { items }\end{array}$ & $\begin{array}{l}\mathrm{N}=77 \text { Madres de } \\
\text { Consulta General privada } \\
\text { (Noruega). } \\
\text { Evaluación Posparto: } \\
2 \text { meses (T1) } \\
4 \text { meses (T4) }\end{array}$ & $\begin{array}{l}\text { Coeficiente Alfa de } \\
\text { Cronbach: } \\
\alpha=0.69\end{array}$ & $\begin{array}{l}-10 \text { ítems que reflejan las } \\
\text { reacciones maternas ante sus } \\
\text { hijos. } \\
\text {-Los ítems tienen una escala de } 5 \\
\text { opciones de respuesta }\end{array}$ & Sin datos \\
\hline
\end{tabular}




\section{CONCLUSIONES}

Evidencia científica ha confirmado la importancia y valía de la inclusión de la salud mental materno-infantil, como parte de la atención a la salud perinatal. Sin embargo, el conocimiento actual describe en su mayoría la realidad de países desarrollados con sistemas de salud y realidades distintas a la latinoamericana. Los avances desiguales en salud mental perinatal entre los países muestran grandes desafíos para Latinoamérica, donde el acceso a los servicios de salud en particular de salud mental y el trabajo multidisciplinario presentan una gran disparidad vinculada a la gran desigualdad social existente en la región. La prevalencia en la región de importantes problemas como la pobreza, la desigualdad en acceso a los sistemas de salud, el embarazo en mujeres adolescentes y elevados índices de violencia e inseguridad social conforman un ambiente de riesgo que afecta en mayor medida a grupos vulnerables como las mujeres y los niños en los países latinoamericanos. Estas condiciones sociales suponen un riesgo para las interacciones tempranas entre las madres y sus recién nacidos, con consecuencias adversas en el bienestar individual del infante a largo plazo y el del contexto familiar con efectos en la salud poblacional.

Algunos de los principales retos visibles en Latinoamérica en cuanto a la salud mental maternoinfantil y en particular en la vinculación temprana madre-bebé son:

- Inclusión de la evaluación y atención a la salud mental materna como prioridad para la promoción de la salud materno-infantil en todos los niveles de los sistemas de salud.

- Diseño y validación de instrumentos y estrategias de evaluación del vínculo materno-infantil adecuados y pertinentes a las características de las poblaciones.

- Identificación de la prevalencia de alteraciones en la vinculación materno-infantil y los factores de riesgo asociados en los diversos grupos sociales, en particular en los más vulnerables (p.e. madres adolescentes, con bajos niveles socioeconómicos y de escolaridad, con trastornos mentales, en áreas rurales y en grupos índigenas y marginales, expuestas a contextos de violencia intrafamiliar y comunitaria).

- Formación de equipos multidisciplinarios de profesionales de la salud en métodos de detección temprana de dificultades en la vinculación materno-infantil y en intervenciones eficientes que disminuyan las consecuencias adversas asociadas.

- Vinculación permanente de la investigación con la profesionalización de la práctica clínica en el campo de salud mental perinatal.

- Promoción de la salud materno-infantil mediante estrategias de salud multidisciplinarias en los diversos niveles de atención sanitaria que disminuyan el efecto de los factores de riesgo específicos de cada población atendida.

Incluir esta temática en la agenda de salud vinculada a la investigación en los países latinoamericanos e impulsar los esfuerzos ya realizados puede ser una inversión estratégica para promover la salud mental de la región. La generación del conocimiento en este campo, permitirá identificar la dimensión de la problemática en los diversos grupos poblacionales, orientada hacia la toma de decisiones en prioridades de salud y la generación de nuevas políticas públicas de salud mental perinatal. El costo de detecciones y atenciones temprana y eficientes de las dificultades vinculares en las diadas madre-bebé es reducido en comparación a los beneficios potenciales que estas iniciativas podrán tener en la mejora de la salud mental materna y el desarrollo psicosocial infantil, aspectos fundamentales del bienestar psicosocial de cualquier región.

\section{REFERENCIAS}

1. Spinner M. Maternal-infant bonding. Can Fam Physician. 1978; 24: 1151-1153.

2. Brockington I. Postpartum psychiatric disorders. Lancet. 2004; 363(9405); 303-310.

3. Brockington I. Maternal rejection of the young child: Present status of the clinical syndrome. Psychopathology. 2011; 44(5): 329-336. DOI: 10.1159/000325058.

4. Hay DF, Pawlby S. Prosocial development in relation to children's and mothers' psychological problems. Child Dev. 2003; 74(5): 1314-1327. DOI:10.1111/1467-8624.00609.

5. Parfitt Y, Ayers S. Postnatal mental health and parenting: the importance of parental anger. Infant Ment Healht J. 2012; 33(4), 400-410. DOI: 10.1002/imhj.21318

6. Waxler E, Thelen K, Muzik M. Maternal perinatal depression-impact on infant and child development. Euro Psychiatry Rev. 2011; 7(1): 41-47.

7. Murray L, Fiori-Cowley A, Hooper R, Cooper P. The impact of postnatal depression and associated adversity on early mother-infant interactions and 
later infant outcome. Child Dev. 1996; 67(5): 25122526. DOI: $10.2307 / 1131637$.

8. Trevarthen C, Aitken KJ. Infant intersubjectivity: research, theory, and clinical applications. J Child Psychol Psychiatry. 2001; 42(1): 3-48. DOI: 10.1111/1469-7610.00701.

9. David H. Born unwanted: Mental health costs and consequences. Am J Orthopsychiatry. 2011; 81(2): 184-192. DOI: 10.1111/j.19390025.2011.01087.x.

10. Schore AN. The effects of early relational trauma on right brain development, affect regulation, and infant mental health. Infant Ment Health J. 2001; 22(1-2): 201-269. DOI: 10.1002/1097-0355(200101/04)22:1<201::AIDIMHJ8>3.0.CO;2-9

11. Tomlinson M, Cooper P, Murray L. The motherinfant relationship and infant attachment in a South African peri-urban settlement. Child Dev. 2005; 76(5): 1044-1054. DOI: $10.1111 / \mathrm{j} .1467-$ 8624.2005.00896.x.

12. Skovgaard AM, Olsen EM, Christiansen E, Houmann T, Landorph SL, Jørgensen T, et al. Predictors (0-10 months) of psychopathology at age $1 \frac{1}{2}$ years $-\mathrm{a}$ general population study in the Copenhagen Child Cohort CCC 2000. J Child Psychol Psychiatry. 2008; 49(5): 553-562. DOI: 10.1111/j.14697610.2007.01860.x.

13. Sundermann JM, DePrince AP. Maltreatment characteristics and emotion regulation (ER) difficulties as predictors of mental health symptoms: results from a community-recruited sample of female adolescents. J Fam Viol. 2015; 30(3): 329-338. DOI: 10.1007/s10896-014-9656-8.

14. Shelton JL, Hoffer TA, Muirhead YE. Behavioral analysis of maternal filicide. Edit. Springer Science+Business Media. New York, USA. 2015.

15. Debowska A, Boduszek D, Dhingra K. Victim, perpretador, and offense characteristics in filicide and filicide-suicide. Agr Viol Behav. 2015; 21(1): 113-124. DOI: 10.1016/j.avb.2015.01.011.

16. Freire C, Figuereido B. Filicídio: Incidência e factores associados. Aná Psicológica. 2006; 24(4): 437-446. DOI: 10.14417/ap.185.

17. Bergman K, Sarkar P, Glover V, O'Connor TG. Maternal prenatal cortisol and infant cognitive development: moderation by infant-mother attachment. Biol Psychiatry. 2010; 67: 1026-1032. DOI:10.1016/j.biopsych.2010.01.002.

18. Broad KD, Curley JP, Keverne EB. Motherinfant bonding and the evolution of mammalian social relationship. Philos Trans R Soc Lond Biol Sci. 2006; 361(1476): 2199-2214. DOI:10.1098/ rstb.2006.1940.

19. Jonas W, Nissen E, Ransjö-Arvidson AB, Mathiesen AS, Uvnäs-Moberg K. Influence of oxytocin or epidural analgesia on personality profile in breastfeeding women: a comparative study. Arch Womens Ment Health. 2008; 11: 335-345. DOI: 10.1007/s00737-008-0027-4.

20. Nissen E, Gustavsson P, Widström A, UvnäsMoberg K. Oxytocin, prolactin, milk production and their relation with personality traits in women after vaginal delivery and Sectio Caesarea. J Psychosom Obstet Gynecol. 1998; 19: 49-58. DOI: 10.3109/01674829809044221.

21. Uvnäs-Moberg K, Widström A, Nissen E, Björvell H. Personality traits in women 4 days postpartum and their correlation with plasma levels of ocytocin and prolactin. J Obstet Gynecol. 1990; 11: 261273. DOI: $10.3109 / 01674829009084422$.

22. Stern D, Bruschweiler-Stern N, Freeland A. The Birth of a Mother. How the Motherhood Experience Changes You Forever. Edit. Basic Books. New York, USA. 1998.

23. Winnicott D. Collected Papers: Through Paediatrics to Psychoanalysis. Edit. Basic Books. New York, USA. 1958.

24. Bowlby J. The making and breaking of affectional bonds. I. Aetiology and psychopathology in the light of attachment theory. An expanded version of the Fiftieth Maudsley Lecture, delivered before the Royal College of Psychiatrists, 19 November 1976. Br J Psychiatry. 1977; 130: 201-210. DOI:10.1192/ bjp.130.3.201.

25. Bowlby J. Attachment and Loss. Volume 1: Attachment. 2nd ed. Edit. Basic Books. New York, USA. 1982.

26. Ainsworth M, Blehar M, Waters E, Wall S. Patterns of Attachment. Ed. Erlbaum. Hillsdale, NJ, USA 1978.

27. Klaus M, Kennell J. Maternal-Infant Bonding: The Impact of Early Separation or Loss on Family Development. Edit. Mosby. St Louis, Missouri. USA. 1976.

28. Taylor A, Atkins R, Kumar R, Adams D, Glover V. A new Mother-to-infant Bonding Scale: links with early maternal mood. Arch Womens Ment Health. 2005 ; 8(1): 45-51. DOI: 10.1007/s00737-0050074-z.

29. Condon J, Corkindale C. The assessment of parent-to-infant attachment: Development of a self-report questionnaire instrument. J Reprod Infant Psychol. 1998; 16(1): 57-76. DOI: 10.1080/02646839808404558.

30. Myers B. Mother-Infant Bonding: the Status of this 
critical-period hypothesis. Developm Review. 1984; 4(3): 240-274. DOI: 10.1016/S0273-2297(84)80007-6.

31. American Psychiatric Association. Diagnostic and Statistical Manual of Mental Disorders. DSM-5. 5th Ed. ed. Edit. APA. Washington, D.C., USA. 2013.

32. World Health Organization. International Statistical Classification of Diseases and Related Health Problems, Tenth Revision (ICD-10). 10th ed. Edit. World Health Organization. Geneva, Switzerland. 1992.

33. Zero-to-Three. Diagnostic Classification: 0-3R: Diagnostic Classification of Mental health and Developmental Disorders of Infancy and Early Childhood: Revised Edition. Edit. Zero To Three Press. Washington, DC, USA. 2005.

34. Klier C, Muzik M. Mother-infant bonding disorders and use of parental bonding questionnaire in clinical practice. World Psychiatry. 2004; 3(2): 102-103. DOI: 10.1007/s00737-006-0150-z.

35. Brockington IF, Aucamp HM, Fraser C. Severe disorders of the mother-infant relationship: definitions and frequency. Arch Womens Ment Health. 2006; 9(5): 243-251. DOI: 10.1007/s00737006-0133-0.

36. Parfitt $\mathrm{Y}$, Ayers S. The effect of postnatal symptoms of post-traumatic stress and depression on the couple's relationship and parent-baby bond. J Reprod Inf Psychol. 2009; 27(2): 127-142. DOI:10.1080/02646830802350831.

37. Stein A, Pearson R, Goodman S, Rapa E, Rahman A, McCallum M, et al. Effects of perinatal mental disorders on the fetus and child. Lancet. 2014; 384(9956): 1800-1819. DOI: 10.1016/S01406736(14)61277-0.

38. Choi H, Yamashita T, Wada Y, Kohigashi M, Mizuhara Y, Nagahara Y, et al. Predictors for exacerbation/improvement of postpartum depression-A focus on anxiety, the mothers' experiences of being cared for by their parents in childhood and borderline personality: a perspective study in Japan. J Affect Disord. 2013; 150(2): 507512. DOI: 10.1016/j.jad.2013.04.051.

39. Della Vedova A, Ducceschi B, Cesana B, Imbasciati A. Maternal bonding and risk of depression in late pregnancy: a survey of Italian nulliparous women. J Reprod Inf Psychol. 2011; 29(3): 208-222. DOI: 10.1080/02646838.2011.592973.

40. Richman A, Miller P, LeVine R. Cultural and educational variations in maternal responsiveness. Developm Psychol. 1992; 28(4): 614-621. DOI: 10.1037/0012-1649.28.4.614.

41. Fuertes M, Faria A, Soares H, Crittenden P. Developmental and evolutionary assumptions in a study about the impact of premature birth and low income on mother-infant interaction. Acta Ethol. 2009; 12(1): 1-11. DOI: 10.1007/s10211-008-0051-4.

42. Barber JS, Axinn WG, Thornton A. Unwanted childbearing, health, and mother-child relationships. J Health Soc Behav. 1999; 40: 231257.

43. Walsh J, Hepper E, Bagge S, Wadephul F, Jomeen J. Maternal-fetal relationships and psychological health: emerging research directions. J Reprod Inf Psychol. 2013; 31(5): 490-499. DOI: 10.1080/02646838.2013.834311.

44. Feldman R. Maternal versus child risk and the development of parent-child and family relationships in five high-risk populations. Dev Psychopathol. 2007; 19: 293-312. DOI: 10.10170S0954579407070150.

45. Feldman R, Greenbaum C, Mayes L, Erlich S. Change in mother-infant interactive behavior: relations to change in the mother, the infant, and the social context. Infant Behav Developm. 1997; 20(2): 151-163. DOI: 10.1080/02646838.2013.834311.

46. Jordan B, Franich-Ray C, Albert N, Anderson V, Northam E, Cochrane A, Menahem S. Early mother-infant relationships after cardiac surgery in infancy. Arch Dis Child. 2014; 99(7): 641-645. DOI: 10.1136/archdischild-2012-303488.

47. Righetti-Veltema M, Conne-Perréard E, Bousquet A, Manzano J. Postpartum depression and motherinfant relationship at 3 months old. J Affect Disord. 2002; 70(3): 291-306. DOI: 10.1016/S01650327(01)00367-6.

48. Robson K, Kumar R. Delayed onset of maternal affection after childbirth. Br J Psychiatry. 1980; 136: 347-353. DOI: 10.1192/bjp.136.4.347.

49. Green J, Richards M, Kitzinger J, Coupland V. Mothers' perceptions of their 6-week-old babies: relationships with antenatal, intrapartum and postnatal factors. Irish J Psychol. 1991; 12(2): 133144. DOI: $10.1080 / 03033910.1991 .10557833$

50. Ahl R, Fausto-Sterlingb A, García-Collc C, Seifer R. Gender and discipline in 5-12-month-old infants: a longitudinal study. Infant Behav Dev. 2013; 36: 199-209. DOI: 10.1016/j.infbeh.2013.01.005.

51. Edhborg M, Nasreen H, Kabir Z. Impact of postpartum depressive and anxiety symptoms on mothers' emotional tie to their infants $2-3$ months postpartum: a population-based study from rural Bangladesh. Arch of Womens Ment Health. 2011; 14(4): 307-316. DOI: 10.1007/s00737-011-0221-7.

52. Chandra P, Satyanarayana V. Gender disadvantage and common mental disorders in women. Int Rev Psychiatry. 2010; 22(5): 513-524. DOI: 
10.3109/09540261.2010.516427.

53. Kim P, Feldman R, Mayes LC, Eicher V, Thompson $\mathrm{N}$, Leckman JF, et al. Breastfeeding, brain activation to own infant cry, and maternal sensitivity. J Child Psychol Psychiatry. 2011; 52(8): 907-915. DOI:10.1111/j.1469-7610.2011.02406.x.

54. Figueiredo $\mathrm{B}$, Dias $\mathrm{CC}$, Brandão $\mathrm{S}$, Canário $\mathrm{C}$, Nunes-Costa, R. Breastfeeding and postpartum depression: state of the art review. J pediatr. 2013; 89(4):332-338. DOI: 10.1016/J.JPED.2012.12.002.

55. Ystrom E. Breastfeeding cessation and symptoms of anxiety and depression: A longitudinal cohort study. Pregn Chilbirth. 2012; 12(36), 1-6. DOI: 10.1186/1471-2393-12-36.

56. Field T. Postpartum depression effects on early interactions, parenting, and safety practices: a review. Infant Behav Dev. 2010; 33: 1-6. DOI: 10.1016/j.infbeh.2009.10.005.

57. Stuebe A, Horton B, Chetwynd E, Watkins S, Grewen K, Meltzer-Brody S. Prevalence and risk factors for early, undesired weaning attributed to lactation dysfunction. J Womens Health. 2014; 23(5): 404-412. DOI: 10.1089/jwh.2013.4506.

58. Gharaibeh M, Hamlan, A. Factors influencing maternal attachment of first-time jordanian mothers. J Res Nurs. 2012; 17(3): 289-303. DOI: $10.1177 / 1744987110395352$.

59. Herguner S, Cicek E, Annagur A, Herguner A, Ors R. Association of delivery type with postpartum depression, perceived social support and maternal attachment. J Psychiatry Neurol Sciences. 2014; 27: 15-20. DOI: 10.5350/DAJPN2014270102.

60. Brockington I, Oates J, George S, Turner D, Vostanis $\mathrm{P}$, Sullivan M, et al. A screening questionnaire for mother-infant bonding disorders. Arch Womens Ment Health. 2001; 3: 133-140. DOI: 10.1007/ s007370170010.

61. Brockington I, Fraser C, Wilson D. The postpartum bonding questionnaire: a validation. Arch Womens Ment Health. 2006; 9(5): 233-242. DOI: 10.1007/ s00737-006-0132-1.

62. Brockington I. The Birmingham Interview for Maternal Mental health. 5th ed. Edit. Eyry Press. Bredenbury, UK. 2006.

63. Siu B, Ip P, Chow H, Kwok SS, Li O, Koo M, et al. Impairment of mother-infant relationship: validation of the Chinese Version of Postpartum Bonding Questionnaire. J Nerv Ment Dis. 2010; 198(3): 174-179. DOI: 10.1097/NMD.0b013e3181d14154.

64. Edhborg M, Matthiesen AS, Lundh W, Widström AM. Some early indicators for depressive symptoms and bonding 2 months postpartum-a study of new mothers and fathers. Arch Womens Ment Health. 2005; 8(4):
221-231. DOI: 10.1007/s00737-005-0097-5.

65. Reck C, Klier CM, Pabst K, Stehle E, Steffenelli U, Struben K, et al. The German version of the Postpartum Bonding Instrument: psychometric properties and association with postpartum depression. Arch Womens Ment Health. 2006; 9(5): 265-271. DOI: 10.1007/s00737-006-0144-x.

66. van Bussel JC, Spitz, B, Demyttenaere K. Three self-report questionnaires of the early mother-toinfant bond: reliability and validity of the dutch version of the MPAS, PBQ and MIBS. Arch Womens Ment Health. 2010; 13(5): 373-384. DOI: 10.1007/s00737-009-0140-z.

67. Naciones Unidas. Objetivos de Desarrollo del Milenio. Informe del 2015. Edit. Naciones Unidas. Nueva York. EEUU. 2015. Recuperado el 23 de Enero del 2016.

68. Boeckel MG, Wagner A, Ritter F, Sohne L, Schein $\mathrm{S}$, Grassi-Oliveira R. Análise fatorial do inventário percepção de vinculação materna. Interam $\mathrm{J}$ Psychol. 2011; 45(3): 439-447.

69. Perrelli JGA, Zambaldi CF, Cantilino A, Sougey EV. Mother-child bonding assessment tools. Rev Paul Pediatr. 2015; 32(3): 257-265. DOI: 10.1590/01030582201432318 .

70. Leckman JF, Mayes L, Feldman R, Evans D, Cohen DJ. The Yale Inventory of Parent Thoughts and Actions. Edit. Yale University. New Haven, USA. 1994.

71. Bernazzani O, Marks M, Bifulco A, Siddle K, Asten P, Conroy S. Assessing psychosocial risk in pregnant/postpartum women using the Contextual Assessment of Maternity Experience (CAME)-recent life adversity, social support and maternal feelings. Soc Psychiatry Psychiatr Epidemiol. 2005; 40(6): 497-508. DOI: 10.1007/s00127-005$0917-\mathrm{y}$.

72. Feldman R. Mother-newborn coding system manual. Tel Aviv, Edit. Bar-Ilan University University Press. Tel-Aviv, Israel. 1998.

73. Kumar R, Hipwell AE. Development of a clinical rating scale to assess mother-infant interaction in a psychiatric mother and baby unit. Br J Psychiatry. 1996; 169(1): 18-26. DOI: 10.1192/bjp.169.1.18.

74. Crittenden PM. Der CARE-Index als Hilfsmittel für Früherkennung, Intervention und Forschung. Frühförderung interdisziplinär (early interdisciplinary intervention). Bindungsorientierte Ansätze in der Praxis der Frühförderung, Special issue. 2005; 24: 99-106.

75. Brockington I, Roper A, Edmunds E, Kaufman C, Meltzer H. A longitudinal psychopathological schedule. Psychol Med. 1992; 22(4): 1035-1043. 
76. Campo-Arias A, Herazo E. Concordancia intra- e interevaluadores. Rev Colomb Psiquiatr. 2010; 39(2): 424-432. DOI: 10.1016/S0034-7450(14)60261-4.

77. Kumar R, Robson K, Smith AM. Development of a self-administered questionnaire to measure maternal adjustment and maternal attitudes during pregnancy and after delivery. J Psychosoma Res. 1984; 28(1): 4351. DOI: 10.1016/0022-3999(84)90039-4.

78. Figueiredo B, Mendonça M, Sousa R. Portuguese validation of the Maternal Adjustment and Maternal Attitudes (MAMA). Psicolog: Saúde Doenças. 2004; 5: 31-51.

79. Koubaa S, Hällström T, Linde'n Hirschberg A. Early Maternal Adjustment in Women with Eating Disorders. Int J Eat Disord. 2008; 41(5): 405-410. DOI: 10.1002/eat.20521.

80. Vivilaki VG, Dafermos V, Gevorgian L, Dimopoulou A, Patelarou E, Bick D, et al. Validation of the Greek maternal adjustment and maternal attitudes scale for assessing early postpartum adjustment. Women Health. 2012; 52(4): 369-390. DOI: 10.1080/03630242.2012.674089.

81. Eganhouse DJ. A comparative study of variables differentiating false labor from early labor. J Perinatol. 1991; 11(3): 249-257.

82. Rouhe H, Salmela-Aro K, Toivanen R, Tokola M, Halmesmäki E, Ryding EL, et al. Group psychoeducation with relaxation for severe fear of childbirth improves maternal adjustment and childbirth experience-- a randomised controlled trial, J Psychosom Obstet Gynecol. 2015; 36(1): 1-9. DOI: 10.3109/0167482X.

83. Müller ME. A questionnaire to measure motherto-infant attachment. J Nurs Meas. 1994; 2(2): 129-141.

84. Ohta N. Development of Maternal Attachment Inventory (MAI) - Revised for Japanese and analysis of factors influencing scores. J Japan Pediatr Soc. 2001; 105(8): 867-875

85. Suetsugu Y, Honjo S, Ikeda M, Kamibeppu K. The Japanese version of the Postpartum Bonding Questionnaire: Examination of the reliability, validity, and scale structure. J Psychosom Res. 2015; 79(1): 55-61. DOI: 10.1016/j.jpsychores.2015.02.0088.

86. Shin H, Kim YH. Maternal Attachment Inventory: psychometric evaluation of the Korean version. J Adv Nurs. 2007; 59(3): 299-307 DOI: 10.1111/j.1365-2648.2007.04322.x.

87. Chen CJ, Sung HC, Chen YC, Chang CY, Lee MS. The development and psychometric evaluation of the Chinese version of the maternal attachment inventory. J Clin Nurs. 2013; 22(19-20): 26872695. doi: 10.1111/jocn.12162.
88. Reay R, Matthey S, Ellwood D, Scott M. Long-term outcomes of participants in a perinatal depression early detection program. J Affect Disord. 2011; 29(1-3): 94-103. DOI: 10.1016/j.jad.2010.07.035.

89. Warner R, Appleby L, Whitton A, Faragher B. Attitudes toward motherhood in postnatal depression: development of the Maternal Attitudes Questionnaire. J Psychosom Res. 1997; 43(4): 351358. DOI: 10.1016/S0022-3999(97)00128-1.

90. Feldstein S, Hane AA, Morrison BM, Huangi KY. Relation of the Postnatal attachment questionnaire to the attachment Q-Set. J Reprod Infant Psychol. 2004; 22: 111-121.

91. Scapesi A, Viterbori P, Sponza S, Zuchinetti P. Assessing mother to-infant attachment: the Italian Adaptation of a self-report questionnaire. J Reprod Infant Psychol. 2004; 22: 99-109.

92. Barnes CR, Adamson-Macedo EN. Perceived Maternal Parenting Self-Efficacy (PMP S-E) tool:development and validation with mothers of hospitalized preterm neonates. J Adv Nurs. 2007; 60(5): 550-560. DOI: 10.1111/j.13652648.2007.04445.x.

93. Nagata M, Nagai Y, Sobajima H, Anto T, Nishide Y, Honjo, S. Maternity blues and attachment to children in mothers of full-term normal infants. Acta Psychiatr Scand. 2000; 101(3): 209-217.

94. Noorlander Y, Bergink V, van den Berg $M$. Perceived and observed mother-child interaction at time of hospitalization and release in postpartum depression and psychosis. Arch Womens Ment Health. 2008; 11(1): 49-56. DOI: 10.1007/s00737008-0217-0.

95. Høivik M, Burkeland N, Linaker O, Berg-Nielsen T. The mother and baby interaction scale: A valid broadband instrument for efficient screening of postpartum interaction? A preliminary validation in a Norwegian community sample. Scand J Caring Sci. 2013; 27(3): 733-739. DOI: 10.1111/j.14716712.2012.01060.x.

96. Haneko H, Honjo S. The psychometric properties and factor estructure of the postpartum bonding questionnaire in Japanese mothers. Psychology. 2014; 5(9): 1135-1142. DOI: 10.4236/psych.2014.59126.

97. García-Esteve L. Torres A, Lasheras G, PalaciosHernández B, Farré-Sender B, Subirà $\mathrm{S}$ et al. Assessment of psychometric properties of the Postpartum Bonding Questionnaire (PBQ) in Spanish mothers. Arch Women Ment Health. 2015; 19(2): 385-394. DOI:10.1007/s00737-015-0589-x.

98. Muzik M, London Bocknek E, Broderick A, Richardson P, Rosenblum KL, Thelen K, et al. Mother-infant bonding impairment across 
the first 6 months postpartum: The primacy of psychopathology in women with childhood abuse and neglect histories. Arch Womens Ment Health. 2013; 16(1): 29-38. DOI: 10.1007/s00737-0120312-0.

99. Tokotzky L. Postpartum maternal sleep, maternal depressive symptoms and self-perceived MotherInfant Emotional relatioship. Behav Sleep Med. 2016; 14(1): 5-22. DOI: 10.1080/15402002.2014940111.

100.Figueiredo B, Marques A, Costa R, Pacheco A, Pais A. Bonding: scale to evaluate parents' motional involvement with their infant. Psychologica. 2005; 40: 133-154.

101.Bienfait M, Maury M, Haquet A, Faillie J, Franc N, Combes $\mathrm{C}$, et al. Pertinence of the self-report motherto-infant bonding scale in the neonatal unit of a materrnity ward. Early Hum Dev. 2011; 87(4): 281287. DOI: 10.1016/j.earlhumdev.2011.01.31.

102. Yoshida K, Yamashita H, Conroy S, Marks M, Kumar C. A Japanese version of mother-to-infant bonding scale: factor structure, longitudinal changes and links with maternal mood during the early postnatal period in Japanese mothers. Arch Womens Ment Health. 2012; 15: 343-352. DOI: 10.1007/s00737-012-0291-1.

103.Robakis TK, Williams KE, Crowe S, Kenna H, Gannon J, Rasgon NL. Optimistic outlook regarding maternity protects against depressive symptoms postpartum. Arch Womens Ment Health. 2015; 18(2): 197-208. DOI: 10.1007/s00737-014-0446-3.

104.Dimitraki M, Tsikouras P, Manav B, Gioka T, Koutlaki N, Zervoudis S, et al. Evaluation of the effect of natural and emotional stress of labor on lactation and breast-feeding. Arch Gynecol Obstet. 2016; 293: 317-328. DOI 10.1007/s00404-015-3783-1.

105.Hall P, Papageorgiou C. Negative thoughts after childbirth: development and preliminary validation of a self-report scale. Depress Anxiety. 2005; 22: 121129. DOI: 10.1002/da.20119. 